\title{
THE PROTECTIVE EFFECT OF INSULIN AGAINST ISOFLURANE AND SEVOFLURANE INDUCED HEPATOCELLULAR APOPTOSIS \\ By
}

KHALDA G. RADWAN ${ }^{1}$, MOSHIRA SAYED ${ }^{*}$, WAEL A. EL-SIORY ${ }^{2}$, MOHAMED A. MAHER ${ }^{1}$, MOHAMED M. HUSSEIN ${ }^{1}$, OLFAT A. HAMMAM ${ }^{3}$, MONA HASSAN ${ }^{4}$, AHMED H. HELMY ${ }^{5}$ and HODA H. OKASHA ${ }^{2}$.

Department of Anesthesia \& SICU ${ }^{1}$, and Department of Anesthesia \& SICU ${ }^{2}$, Faculty of medicine Cairo University, Giza and Department of Pathology ${ }^{3}$, Department of Clinical Chemistry ${ }^{4}$ and Department of General surgery ${ }^{5}$, Theodor Bilharz Research Institute ${ }^{1,3,4,5}$, Imbaba B.O. Box 30, Giza12411, Egypt

$\left({ }^{*}\right.$ Correspondence: Moshira.amer83@gmail.com.; Mobile: 01227206511)

\begin{abstract}
Several studies deducted that inhalational anesthetics induce apoptosis in human cells. Insulin is believed to have an antiapoptotic action so it is widely used as cardioprotective agent against ischemic reperfusion injuries. This study compared the apoptotic effect of inhalational anesthetics and figuring out the antiapoptotic effect of insulin against perioperative induced hepatocellular apoptosis using immune histochemical study of liver biopsy.

Eighty (ASA I) patients scheduled for laparoscopic cholecystectomy were randomly allocated into 4 groups (20 patients each). Two groups were anesthetized using isoflurane and the other two were anesthetized using sevoflurane. The control groups (IC, SC) received normal saline and the insulin groups (II, SI) received glucose, insulin and potassium (GIK) infusion. Infusions were given 2 hours before induction of anesthesia. Liver biopsy was taken before the umbilical port closure. In liver biopsy Caspase 3, 7, 9 and Akt activity were evaluated. Liver function tests were estimated before infusion and one day after surgery. Serum K and blood glucose levels were closely monitored all through the study.

The results showed that in the isoflurane groups, the percentage of caspase 3 and 7 positive cases decreased while Akt positive cases increased significantly in II compared to IC respectively $(\mathrm{p}<0.05)$. In the sevoflurane groups, the percentage of caspase 3 positive cases decreased significantly in SI compared to SC group ( $\mathrm{p}<0.05)$.
\end{abstract}

Key words: Protective, perioperative, hepatocellular, apoptosis, insulin

\section{Introduction}

Apoptosis is an ordered and orchestrated cellular death that occurs physiologically as part of organ maturation and homeostasis (Elmore, 2007), in addition it is induced in some pathological conditions i.e. exposure to drugs and pathogens (Nikitakis et al. 2004). Cellular apoptosis is generally executed through activation of a group of intracellular enzymes called caspases (Olijnyk, 2007). Caspases are activated either extrinsically by binding of ligands to specialized receptor on the cell membrane called death receptors $(\mathrm{Li}, 2008)$ or they were activated intrinsically by mitochondrial release of cytochrome c (Dipak et al, 2012).

Surgical trauma leads to a complex systemic response including sympathetic nervous system activation, endocrine response, inflam- matory and immunological disturbances. Surgical trauma includes direct tissue damage and other factors including: exposure to anesthetics, blood loss and/or blood transfusion, hypothermia, immobility, induced general or local ischemia in some procedures and reperfusion injury. All these factors induced the cellular apoptosis (Kosela et al, 2016).

The commonly used inhalational anesthetics; isoflurane and sevoflurane are believed to induce apoptosis in various types of tissues and cells including liver cells, hippocampal slices, lymphocytes and developing neurons with different potencies (Loop and Dovi-Akue, 2005; Dong et al, 2009; Yingxian et al, 2017). Many theories were proposed for the mechanism by which they induce apoptosis. The latest was either that 
they increased intracellular calcium concentration disrupting mitochondrial membrane (Zhang GH et al, 2008) or production of high levels of reactive oxygen species (ROS) during their oxidative metabolism in hepatocytes by the cytochrome P450 enzymes. Both scenarios lead to mitochondrial membrane rupture and release of cytochrome c that initiates caspase activation, which executes apoptosis (Wang, 2014).

Insulin is widely used as an antiapoptotic agent in myocardial protective strategies by various GIK infusion regimens (Yosuke et $a l, 2017)$. Insulin exerts its antiapoptotic action through activation of serine threonine kinase (Akt) which is an enzyme nearly found in all human cells (Richard et al, 2014). Akt then mediates binding of hexokinase enzyme to the mitochondria (Chiara et $a l, 2008)$. The binding of intracellular hexokinase to mitochondrial membranes lead to a number of antiapoptotic events: 1- It suppressed free radical generation and hence reduces oxidative damage to the cell (DaSilva et al, 2004). 2- It inhibited the release of cytochrome c from the mitochondria into the cytosol (Pastorino et al, 2002). 3- It might prevent the opening of mitochondrial permeability transition pores preventing mitochondrial swelling and apoptosis (Chiara et al, 2008, Aikawa et al, 2000).

Up to the knowledge of the authors, insulin's antiapoptotic action was only studied as a cardioprotective agent in acute coronary syndromes and in cardiac surgeries (Richard et al, 2014; Yosuke et al, 2017). These studies assessed apoptosis either using human serum markers or in vitro on animal cardio-myocytes decreasing their specificity and clinical relevance respectively (Osman et al, 2012).

In the current study, we investigated the apoptotic effect of the two commonly used inhalational anesthetics in the liver and we intended to explore a new clinical application of the antiapoptotic protective effect of insulin on liver cells. To be specific we assessed apoptosis by evaluating Akt, caspase
3, 7 \& 9 activities immune histochemically in liver biopsy taken at the end of laparoscopic cholecystectomy in non-diabetic patients.

\section{Materials and Methods:}

This study was designed to be: single center prospective randomized controlled single blinded clinical trial. The protocol was approved by the research ethics committee in Theodore Bilharz Research Institute (TBRI).

Sample size: The study was designed as a pilot study and enrolled 80 ASA I patients undergoing laparoscopic cholecystectomy.

Patients selection: Eight ASA I patients scheduled for laparoscopic cholecystectomy, aged between 25 to 55 years with body mass index (BMI) from 25-35 were enrolled in the study. All patients were randomly allocated into four groups (20 patients each). Two groups were anesthetized using isoflurane and were randomly allocated into control group IC $(n=20)$ receiving normal saline infusion and insulin group II $(n=20)$ receiving GIK regimen. The other two groups were anesthetized using sevoflurane and were randomly allocated into control group SC $(n=20)$ receiving normal saline infusion and insulin group SI $(n=20)$ receiving GIK regimen.

Exclusion criteria: The exclusion criteria included (1) pre-diabetic patients hemoglobin A1c > 6.5\%; (2) acute cholecystitis (3) pregnancy and lactation; (4) fever (5) need for intraoperative blood transfusion; (6) intraoperative shifting to open cholecystectomy; (7) fatty liver; (8) and/or drug abusers.

Preoperative preparation: Two hours before surgery, intra-venous (IV) $20 \mathrm{G}$ cannula was inserted in each patient. Blood samples were withdrawn for estimation of laboratory investigations. Then, the fluid administration started by normal saline in GIC \& GSC while GII \& GSI received GIK infusion which was composed of $50 \mathrm{ml}$ of $10 \%$ dextrose, $10 \mathrm{mEq}$ of potassium, and $10 \mathrm{IU}$ of regular insulin (Stefano Di Marco. et al 2010). All fluids were infused over 60 minutes to be finished $1 \mathrm{hr}$ before starting 
the operation. All patients did not receive any premedication or sedation before induction of anesthesia.

Induction and maintenance of general anesthesia: Anesthesia was induced with 1.5 to $2.0 \mathrm{mg} / \mathrm{kg}$ propofol (B/BRAUN, Egypt) and $2 \mu \mathrm{g} / \mathrm{kg}$ fentanyl (Sunny Pharmaceutical, Egypt). Neuromuscular blockade was achieved by $0.5 \mathrm{mg} / \mathrm{kg}$ atracurium (Sunny Pharmaceutical, Egypt) followed by tracheal intubation. Standard anesthesia care was provided, including fluid management with ringer acetate and routine monitoring of electrocardiogram, non invasive arterial blood pressure, end tidal capnography, anesthesia gas analyzer, bispectral index (BIS) and oxygen saturation. Inhalational anesthetic end tidal concentration was adjusted around 1 MAC to maintain BIS values 40 to 60. Hemodynamic measurements (MAP, HR $\& \mathrm{SpO}_{2}$ ) were recorded pre-induction, post induction, every 5 minutes till the end of surgery, after recovery and every 4 hours postoperatively for 24 hours.

Blood sampling: Liver function tests including serum alanin amino-transferase (ALT), aspartate amino-transferase (AST), albumin (Alb) and total bilirubin were estimated; before starting fluid infusion and one day postoperatively. Serum $\mathrm{K}^{+}$was checked; before starting fluid infusion and immediately post-operative. Blood glucose level was closely monitored using capillary measurement before and after finishing GIK, directly after induction of anesthesia then every 30 minutes till end of surgery. Postoperatively it was measured every $2 \mathrm{Hrs}$ "in the first 6 Hrs" then every 6 Hrs till the timing of the 24 post-operative sample.

Liver biopsy collection and handling: For all patients liver biopsy was taken at the end of surgery before the umbilical port closure. Both duration of inhalational anesthetic exposure and time from induction of anesthesia to taking the biopsy were recorded. The pathologist was blinded to the group types.

Histopathology: 1- Liver biopsies were fixed immediately after removal in $10 \%$ for- malin buffered with $0.1 \mathrm{M}$ phosphate buffer. 2- The specimens were trimmed and processed into paraffin wax according to standard histopathological laboratory methods. 3Sections were prepared from the paraffin blocks, cut at $5 \mu \mathrm{m}$ in thickness. 4- All slides were treated with 3amino-propyl-triethoxysilane (3APTES/SIGMA-A-3648). These slides were used instead of the ordinary albumenized slides to minimize staining artifacts and for better fixation of the sections on the slides.

Immune histochemical (IHC) technique: Expression of Akt, caspase 3, 7 and 9 proteins were evaluated using a commercial Akt (A-11): sc-377457, caspase 3 (3CSP03): sc56046c, caspase 7 p20 (B-5): sc-28295 and caspase 9 (96.1.23): sc-56076, monoclonal antibodies purchased from Santa Cruz biotechnology company, USA. Deparaffinization, hydration of the slides, and blocking with pre-antibody solution $(20 \mathrm{~min})$ where performed in Dako PT Link (Code PT100/ PT101). Then, a protocol template was created. Staining steps and incubation times were pre-programmed into Autostainer Link software (Dako Autostainer). These were diluting anti-kt1, anti-caspase 3, anti-caspase 7 and anti-caspase 9 primary antibodies (1:150 each for 20min at room temp); applying Poly-HRP anti-rabbit IgG (20 min); applying DAB (20 min, Sigma Fast DAB tablets, Sigma-Aldrich, St. Louis MO); counter staining with EnVision FLEX hematoxylin (5 min); and dehydration, clearing, mounting, and covering.

Immune histochemical scoring of antibodies: Expression of caspase 3, 7, 9 \& Akt were scored by a semiquantitive technique, and all markers are cytoplasmic brown stain, sections were examined under Zeiss light microscopy at $x 40$, in ten microscopic fields. The mean of the percentage of the positively stained cells was calculated from their mean values $=>20 \%$ activity was considered reactive (Shen et al, 2004).

Primary outcome: The evaluation of the apoptotic changes of isoflurane and sevoflu- 
rane by measuring the percentage of caspase 3 positive cases.

Secondary outcomes: Evaluating insulin's antiapoptotic effect against perioperative hepatocellular apoptosis and to determine if this effect was through Akt inhibitory pathway or not. Also assessing the safety of GIK regimen in non diabetics and to figure out if the insulin has a clinical impact or not.

Statistical analysis: Data were analyzed by Chi square to compare between categorical. Due to normality test, comparison between variables in two groups was performed using either unpaired t test or Mann Whitney test whenever appropriated. In normal data, intra-group comparison (within group comparison) between mean values of variables measured at different times (pre-operative, intra-operative, recovery \& post-operative) was performed using repeated measures ANOVA followed by Bonferroni test if sig nificant results were recorded, expressed as mean \pm SD or number $(\%)$.

\section{Results}

The results were shown in tables $(1,2,3$ \& 4 ) and in figures (1, 2, 3, 4 \& 5).

Eighty four eligible patients were randomly allocated into four groups; isoflurane control (GIC) $n=21$, isoflurane insulin GII) $n=22$ sevoflurane control (GSC) $n=21$ \& sevoflurane insulin (GSI) $n=20$. Eighty patients completed the trial from February 2013 to March 2014. One patient was excluded from isoflurane control group due blood loss > $500 \mathrm{ml}$ which required intraoperative blood transfusion. Two patients were excluded from isoflurane insulin group due to intraoperative shifting to open cholecystectomy was technical surgical difficulties. One patient was excluded from sevoflurane control as the liver discovered to be fatty intraoperative.

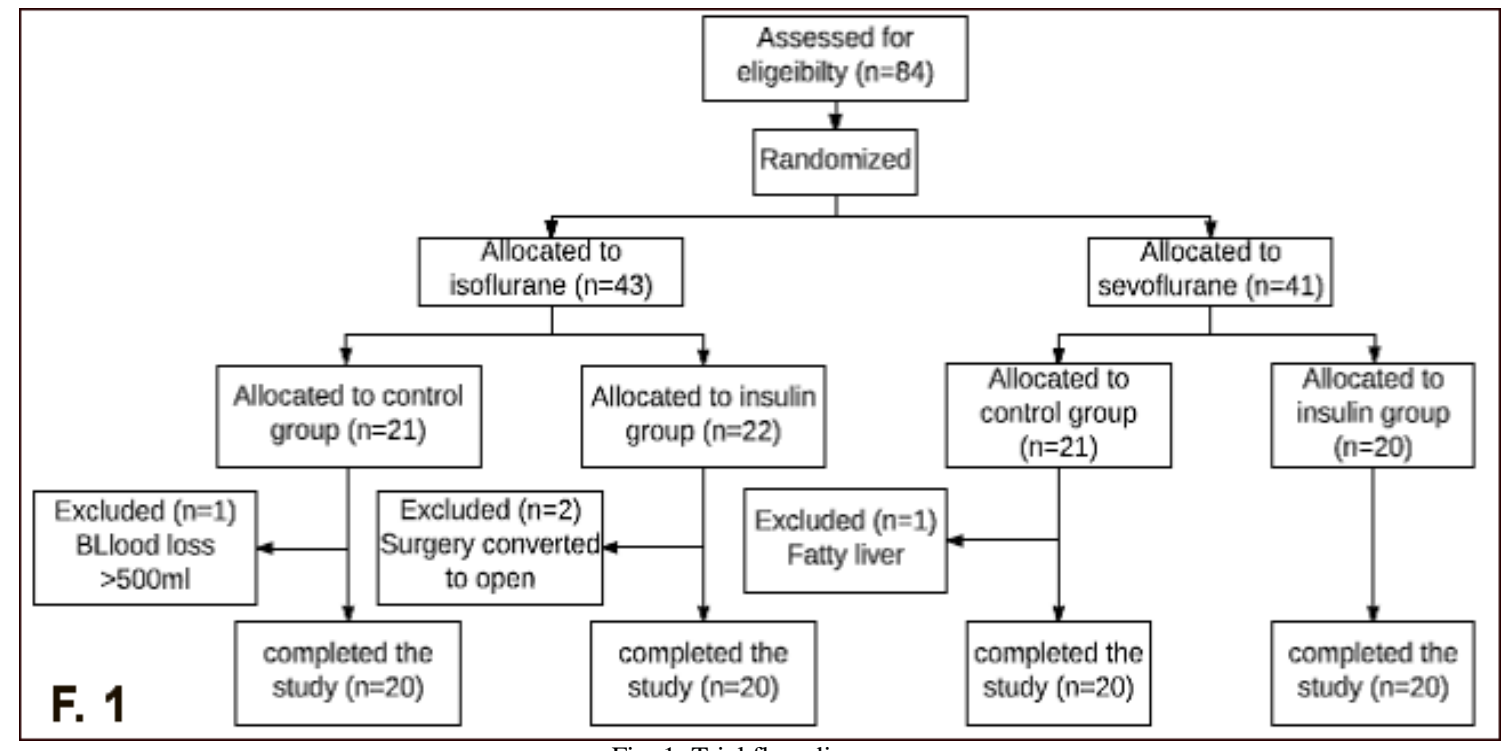

All patients were females without significant difference as to age, BMI, duration of inhalational anesthetics exposure and time from induction of anesthesia till time of biopsy taking. No significant difference was

between insulin and control and all groups concerning hemodynamic variables, oxygen saturation, core temperature and end tidal capnography neither intraoperative nor postoperatively.

Table 1: Baseline characteristics of different studied groups.

\begin{tabular}{|l|l|l|l|l|}
\hline & $\mathrm{IC}(\mathrm{n}=20)$ & $\mathrm{II}(\mathrm{n}=20)$ & $\mathrm{SC}(\mathrm{n}=20)$ & $\mathrm{SI}(\mathrm{n}=20)$ \\
\hline Age $($ years) & $33.4 \pm 6.1$ & $36 \pm 5.8$ & $36.2 \pm 8$ & $37.5 \pm 7.8$ \\
\hline Gender (female) & $20(100 \%)$ & $20(100 \%)$ & $20(100 \%)$ & $20(100 \%)$ \\
\hline BMI $\left(\mathrm{Kg} / \mathrm{m}^{2}\right)$ & $28.7 \pm 3.6$ & $29.6 \pm 3.5$ & $28.9 \pm 3.2$ & $28.7 \pm 3.4$ \\
\hline Duration of anesthesia (min) & $69.5 \pm 4.9$ & $69.1 \pm 5.3$ & $69.2 \pm 3.2$ & $69.3 \pm 4.1$ \\
\hline Time to biopsy (min) & $61.5 \pm 5$ & $60.6 \pm 5.4$ & $60.3 \pm 5.1$ & $60.2 \pm 5$ \\
\hline
\end{tabular}


Regarding liver biopsy; percentage of caspase 3 positive cases in GSC was lower than GIC. Also, percentage of caspase $3 \& 7$ positive cases in GII was lower than GIC and percentage of Akt positive in GII was higher than GIC. In GSI, caspase 3 was the only marker that showed decrease in positive cases in GSI compared to GSC.

Percentage of positive caspase 3 cases in GII (30\%) was significantly lower than in GIC $(80 \%) \mathrm{p}<0.05$. Percentage of positive caspase 7 cases in GII (10\%) was significantly lower than GIC $(90 \%) \mathrm{p}<0.05$ (Fig. 3). Percentage of positive caspase 9 cases in GII $(15 \%)$ was lower than GIC $(25 \%)$ but without significant difference. Percentage of positive Akt cases in GII (70\%) was significantly higher than GIC $(35 \%) \mathrm{p}<0.05$.

Percentage of positive caspase 3 cases in GSI $(35 \%)$ was significantly lower than in GSC (75\%) $\mathrm{p}<0.05$ (Fig.2). Also, the percentage of positive caspase 7 cases in GSI $(10 \%)$ was lower than GSC (30\%) but without significant difference (Fig.3). However, caspase 7 showed the percentage of its positive cases in GSC (30\%) was significantly lower than GIC (90\%) $\mathrm{p}<0.05$.Caspase 9 showed same percentage of positive cases in GSC \& GSI (20\%). Percentage of positive Akt cases in GSI (60\%) was higher than GSC (40\%) without significant difference between them.

Table 2: Percentage of positive cases of caspase 3, 7,9 and Akt activity in control and insulin groups

\begin{tabular}{|l|c|c|c|c|}
\hline Marker & IC $(\mathrm{n}=20)$ & $\mathrm{II}(\mathrm{n}=20)$ & $\mathrm{SC}(\mathrm{n}=20)$ & $\mathrm{SI}(\mathrm{n}=20)$ \\
\hline Caspase 3 & $80 \%$ & $30 \%^{*}$ & $75 \%$ & $35 \% *$ \\
\hline Caspase 7 & $90 \%$ & $10 \%^{*}$ & $30 \%^{\circ}$ & $10 \%$ \\
\hline Caspase 9 & $25 \%$ & $15 \%$ & $20 \%$ & $20 \%$ \\
\hline Akt & $35 \%$ & $70 \%^{*}$ & $40 \%$ & $60 \%$ \\
\hline
\end{tabular}

Insulin did not show impact on biochemical markers, all values were within normal range.

Table 3: Liver function tests in insulin and control groups measured preoperatively and 24 hours postoperative.

\begin{tabular}{|l|l|l|l|l|l|}
\hline Liver function test & IC $(\mathrm{n}=20)$ & $\mathrm{II}(\mathrm{n}=20)$ & $\mathrm{SC}(\mathrm{n}=20)$ & $\mathrm{SI}(\mathrm{n}=20)$ \\
\hline \multirow{2}{*}{ ALT IU/L } & preoperative & $22.3 \pm 12.1$ & $20 \pm 8$ & $19.3 \pm 11.9$ & $22.5 \pm 6.6$ \\
\cline { 2 - 6 } & $24 \mathrm{hs}$ postoperative & $27.6 \pm 9.9^{*}$ & $28.4 \pm 13^{*}$ & $24.5 \pm 10.7^{*}$ & $22.9 \pm 5.6$ \\
\hline \multirow{2}{*}{ AST IU/L } & preoperative & $22.9 \pm 6.7$ & $21.7 \pm 8.1$ & $19.9 \pm 5.5$ & $23.6 \pm 7.8$ \\
\cline { 2 - 6 } & $24 \mathrm{hs}$ postoperative & $26.7 \pm 6.6^{*}$ & $28.7 \pm 10.6^{*}$ & $23.1 \pm 7.4^{*}$ & $22.9 \pm 6.2$ \\
\hline \multirow{2}{*}{ T. bilirubin mg/dl $\mathrm{mg} / \mathrm{dl}$} & preoperative & $4 \pm 0.3$ & $4.1 \pm 0.2$ & $4.1 \pm 0.3$ & $4.1 \pm 0.3$ \\
\cline { 2 - 6 } & 24hs postoperative & $3.8 \pm 0.2^{*}$ & $3.8 \pm 0.3^{*}$ & $3.8 \pm 0.3 *$ & $3.9 \pm 0.2^{*}$ \\
\cline { 2 - 6 } & preoperative & $0.6 \pm 0.2$ & $0.5 \pm 0.2$ & $0.6 \pm 0.2$ & $0.6 \pm 0.2$ \\
\cline { 2 - 6 } & 24hs postoperative & $0.6 \pm 0.2$ & $0.5 \pm 0.7$ & $0.7 \pm 0.2$ & $0.5 \pm 0.1^{*}$ \\
\hline
\end{tabular}

ALT: Alanin amino-transferase., $* \mathrm{P}<0.05$ relative to preoperative level. AST: Aspartate amino-transferase, Alb: Albumin, T. bilirubin: Total bilirubin.

The GIK regimen was safe in non diabetic patients, all serum $\mathrm{K}$ were within normal range despite significant decrease in GII \& GIC postoperatively $(3.8 \pm 0.3 ; 3.91 \pm 0.3)$ than preoperative value $(4.00 \pm 0.3 ; 4.2 \pm 0.3)$ respectively. As to sevoflurane showed significant decrease in GSC postoperative value (3.9 \pm 0.3$)$ compared to preoperative value $(4.2 \pm 0.4)(\mathrm{p}<0.05)$. All the blood glucose measurements were within normal range.
But,. in GII \& GSI, intraoperative, recovery and postoperative mean values were significantly higher than preoperative mean values $(\mathrm{p}<0.05)$, in GIC intraoperative, recovery and postoperative mean values were significantly lower than preoperative mean values $(\mathrm{p}<0.05)$ and in GSC intraoperative, recovery and postoperative mean values were significantly higher than preoperative mean value $(\mathrm{p}<0.05)$.

Table 4:Mean random blood sugar (RBS) measurements in insulin and control groups.

\begin{tabular}{|c|c|c|c|c|}
\hline RBS (mg/dl) & IC $(\mathrm{n}=20)$ & II $(\mathrm{n}=20)$ & $\mathrm{SC}(\mathrm{n}=20)$ & $\mathrm{SI}(\mathrm{n}=20)$ \\
\hline Preoperative & $83.4 \pm 7.8$ & $76 \pm 6$ & $74.3 \pm 4$ & $75.3 \pm 5.2$ \\
\hline Intra-operative & $79.5 \pm 3.9 *$ & $88.7 \pm 3.3^{*}$ & $79.5 \pm 2.3^{*}$ & $86.5 \pm 5.1^{*}$ \\
\hline Recovery & $76.3 \pm 4.7 *$ & $82.6 \pm 4.4 *$ & $77.8 \pm 4.7 *$ & $81.8 \pm 6.6 *$ \\
\hline Post-operative & $80.1 \pm 3.3 *$ & $79 \pm 2.5 *$ & $78 \pm 1.9 *$ & $80.3 \pm 4.7 *$ \\
\hline
\end{tabular}




\section{Discussion}

The current study showed that, isoflurane induced more hepatocellular apoptosis than sevoflurane and that insulin had antiapoptotic effect against perioperative hepatocellular apoptosis through activation of Akt inhibitory pathway. Insulin's antiapoptotic effect was more prominent with isoflurane than sevoflurane. Despite its antiapoptotic action, insulin did not show clinical protective impact on postoperative liver biochemical markers. Moreover, our recommended GIK infusion regimen was well tolerated by non diabetic patients.

These findings were shown in the liver biopsy as caspase 3 activities was higher in isoflurane control group than sevoflurane control group. Also, caspase $3 \& 7$ activities were lower in insulin groups compared to control groups. Also, it was found that insulin exerted its antiapoptotic action through Akt inhibitory pathway as it was higher in insulin groups compared to control groups.

In the current study, laparoscopic cholecystectomy surgery was chose due to the feasibility of obtaining liver biopsy during the procedure. The resultant hepatic apoptosis might have occurred due to either surgical stress response or due to inhalational anesthetic exposure and since there is no specific way to identify the actual cause of apoptosis, we hypothesized that the use of two different inhalational anesthetics and comparing the resultant apoptotic changes while all the patients are subjected to almost similar durations of anesthetic exposure and to the same surgical stress response would enable us to differentiate the cause of apoptosis. So, it could be assumed that the apoptotic changes in the liver were due to anesthetic exposure and not due to surgery as there was variation in the apoptotic changes between both GIC and GSC.

There were numerous studies investigated the apoptotic effect of the inhalational anesthetics, most of these studies were either in vitro (Osman et al, 2012) or on animals (Ruxanda et al, 2016). So the present study, might be the first to evaluate the hepatocellular apoptotic effect of inhalational anesthetics in vivo and aiming to reach a conclusion about which one is safer. It was shown in our results that isoflurane induced more apoptotic changes than sevoflurane. This agreed with Ruxanda et al. (2016) who concluded that: isoflurane triggered apoptosis in a relatively small number of hepatocytes, whereas sevoflurane did not. Also, Yang, et al, (2008) suggested that Isoflurane has greater potency than sevoflurane and desflurane to cause calcium release from the endoplasmic reticulum and inducing apoptosis through elevated levels of caspase 3 and annexin v. On the other hand, George et al. (2011) suggested that in neonatal mice, equipotent doses of the three commonly used inhaled anesthetics "isoflurane, sevoflurane and desflurane" demonstrated similar neuro apoptotic profiles. Also, Osman et al. (2012) suggested that Isoflurane appeared to have less apoptotic effect than sevoflurane

Up to the knowledge of the authors, the current study is the first to investigate the use of insulin as hepatoprotective drug beside its established use as cardioprotective agent in ischemic heart disease (Sack and Yellon, 2003; Mellbin and Rydén, 2012; Sato et al, 2014), ischemic pre-conditioning (Di Marco et al, 2010), in cardiac surgeries (Jovic et al, 2009) and in septic shock (WonYoung et al, 2016). Moreover, nearly all studies which investigated apoptosis were done either on animal models (Raphael et al, 2006, George et al, 2011) or in cell culture lines (Yongxing et al, 2014; Kuriakose et al, 2014; Weber et al, 2015).

Apoptosis (and not necrosis) is the main mechanism of liver injury, especially drug related or after viral injuries. Hepatic cells contained the highest concentration of cytochrome P450 (CYP) which metabolized halogenated anesthetics beside many drugs (Wang, 2014). This explains the hepatocytes' susceptibility to damage and apoptosis upon exposure to inhalational anesthetics 
which was shown in several studies (Cohen Jessica et al, 2010; Ruxanda et al, 2016, Yingxian et al, 2017). This may explain the mild elevation of liver enzymes noticed postoperatively in study groups.

There were many methods of insulin administration, on reviewing the literature but the GIK regimen used by Di Marco et al. (2009) which showed cardioprotective effect with the highest safety profile was selected. This was revealed in our study as we found no deviations in serum $\mathrm{K}^{+}$and blood glucose levels outside the normal clinical ranges after its administration. Though it caused mild elevations in blood glucose levels intra and postoperatively we can still suggest that it is safe to be used in non-diabetics patients. There were other studied GIK regimens with less patient tolerance for example as Jovic et al. (2009). They used GIK regimen 30\% glucose, insulin $2 \mathrm{IU} / \mathrm{kg}, \mathrm{K}^{+} 80 \mathrm{mmol} / \mathrm{l}$ at 1 $\mathrm{ml} / \mathrm{kg} / \mathrm{h}$ \& GIK regimen $10 \%$ glucose, insulin $32 \mathrm{IU}, \mathrm{K}^{+} 80 \mathrm{mmol} / \mathrm{l}$ at $1 \mathrm{ml} / \mathrm{kg} / \mathrm{h}$. They found that it caused variable rates of both hypoglycemia and hyperglycemia outside normal ranges though being both cardioprotective.

\section{Conclusion and Limitations}

Isoflurane induced more apoptotic changes than sevoflurane and insulin has a protective effect against perioperative induced hepatocellular apoptosis. This offers new field for further researches evaluating the use of insulin in other conditions where apoptosis plays important role in its pathogenesis i.e. viral hepatitis, liver cell failure and reperfusion injury in liver transplantation.

Some limitations in the present study bear discussion. First, although insulin proved to have protective effect on the liver yet its effect didn't show clinical impact as liver enzymes was elevated in studied groups. This might be attributed to relative short duration of anesthetic exposure or the relatively low dose of insulin so we can recommend studying the insulin's protective effect in more prolonged surgical procedures. Second patients were all females. This was not intend- ed but the eligible patients meeting the criteria of this study were only females due to relative rarity of male patients scheduled for laparoscopic cholecystectomy in the present institute at that time. So, gender discrepancy might have affected the results.

Funding and support: The work was supported by Theodor Bilharz Research Institute as a project number (104 A).

Registration of clinical trial: the trial was registered and allocated in the Australian New Zealand Clinical Trials Registry (ANZ-CTR). The ACTRN: ACTRN 12616000556448. Web address of the trial: http:// www. ANZCTR.org.au/ACTRN12616000556448.as px: Date submitted: 7/12/2015. Date registered: 29/04/2016.

Conflicts of interests: All the authors have no conflicts of interests to declare in this work.

\section{References}

Aikawa, R, Nawano, M, Gu, Y, et al, 2000: Insulin prevents cardiomyocytes from oxidative stress induced apoptosis through activation of PI3 kinase/Akt. Circulation 102:2873-9.

Chiara, F, Castellaro, D, Marin, O, et al, 2008: Hexokinase II detachment from mitochondria triggers apoptosis through the permeability transition pore independent of voltagedependent anion channels. PLoS One 3:1-11.

Cohen Jessica, I, Sanjoy, R, Megan, MR, et al, 2010; Complement and Alcoholic Liver Disease: Role of C1q in the Pathogenesis of Ethanol-Induced Liver Injury in Mice. Gastroenterology 139:664-674.

Da-Silva, WS, Gomez-Puyou A, de GomezPuyou MT, et al, 2004: Mitochondrial bound hexokinase activity as a preventive antioxidant defense: steady-state ADP formation as a regulatory mechanism of membrane potential and reactive oxygen species generation in mitochondria. J. Biol. Chem. 279:39846-55.

Di Marco, S, Beatrice Boldrini, B, Umberto Conti, U, et al, 2010: Effects of GIK (glucoseinsulin-potassium) on stress induced myocardial ischemia. Clin. Sci. 119:37-44.

Dipak, D, Ghatage, S, Gosavi, R, Ganvir, SM, et al, 2012: Apoptosis: Molecular mechanism. J. Orofacial Sci. 4:103-7.

Dong, G, Zhang, B, Zhang, GH, et al, 2009: The common inhalational anesthetic sevoflurane 
induces apoptosis and increases $\beta$-amyloid protein levels. Arch. Neurol. 66:620-31.

Elmore S. 2007; Apoptosis: a review of programmed cell death. Toxicol. Pathol. 35:495516.

George, K, Istaphanous, J, Howard, X, Xinyu $\mathrm{N}$, et al, 2011: Comparison of the neuroapoptotic properties of equipotent anesthetic concentrations of desflurane, isoflurane, or sevoflurane in neonatal mice. Anesthesiology 114:578-87.

Huafeng Wei. 2011: The Role of Calcium Dysregulation in Anesthetic-Mediated Neurotoxicity. Anesth. Analg. 113:972-4.

Jovic, M, Gradinac, S, Lausevic-Vuk, L, et al, 2009: Preconditioning with glucose-insulin-potassium solution and restoration of myocardial function during coronary surgery. Gen. Physiol. Biophys. 28:262-70.

Kosela, J, Rusakb, M, Golembiewski, L, et al, 2016; Total knee replacement induces peripheral blood lymphocytes apoptosis and it is not prevented by regional anesthesia. Rev Bras Anestesiol. 66:133-139.

Kuriakose, GC, Singh, S, Rajvanshi, PK, et $a l, 2014$; In vitro cytotoxicity and apoptosis induction in human cancer cells by culture extract of an Endophytic Fusarium solani strain isolated from Datura metel L. Pharm. Anal. Acta. 5:4-12. Li, JY, 2008: Caspases in apoptosis and beyond. Oncogene.27: 6194-7206.

Loop, T, Dovi-Akue, D, 2005; Volatile Anesthetics Induce Caspase-dependent, Mitochondria-mediated Apoptosis in Human T Lymphocytes In Vitro. Anesthesiology 102: 1147-57.

Mellbin, L, Rydén, L, 2012: Evidence for a beneficial effect of glucose-insulin-potassium in patients with acute coronary syndromes. Did the immediate trial solve an unanswered question? Expert Rev. Cardiovasc. Ther. 10:1097-9.

Nikitakis NG, Sauk JJ, Papanicolaou SI. 2004; The role of apoptosis in oral disease: Mechanisms; aberrations in neoplastic, autoimmune, infectious, hematologic, and developmental diseases; and therapeutic opportunities. Oral Surg. Oral Med. Oral Pathol. Oral Radiol. Endod. 97:476-90.

Olijnyk, AM, 2007; Apoptosis: Rules, Regulation and P53. Biology 508:1-29.

Osman, ES, Khafagy, HF, Samhan, YM, et al, 2012: In vivo effects of different anesthetic agents on apoptosis. Korean J. Anesthesiol. 63:
18-24.

Pastorino, JG, Shulga, N, Hoek, JB, 2002: Mitochondrial binding of hexokinase II inhibits Bax-induced cytochrome c release and apoptosis. J. Biol. Chem. 277:7610-8.

Raphael, J, Abedat, S, Rivo, J, et al, 2006: Volatile anesthetic preconditioning attenuates myocardial apoptosis in rabbits after regional ischemia and reperfusion via Akt signaling and modulation of Bcl-2 family proteins. J. Pharmacol. Exp. Ther. 318:186-94.

Richard, WA, Mackenzie, Elliott, BT, 2014: Akt/PKB activation and insulin signaling: A novel insulin signaling pathway in the treatment of type 2 diabetes. Dove Press Diabetes, Metabolic Syndrome and Obesity: Targets Ther. 7: 55-64.

Ruxandaa, F, Florin, A, Galb, C, Ratiuc, A, et al, 2016: Comparative immune-histochemical assessment of the effect of repetitive anesthesia with isoflurane and sevoflurane on rat liver. Rev. Bras. Anestesiol. 66:465-9.

Sack, MN, Yellon, DM, 2003: Insulin therapy as an adjunct to reperfusion after acute coronary ischemia: a proposed direct myocardial cell survival effect independent of metabolic modulation. J. Am. Coll. Cardiol. 41:1404-7.

Sato, T, Sato, H, Oguchi, T, et al, 2014: Insulin preconditioning elevates P-Akt and cardiac contractility after reperfusion in the isolated ischemic rat heart. BioMed. Res. Inter. Vol. 1-7.

Shen, HW, Yi, Y, Wangm XM, et al, 2004: Expression of caspase-3 and Bcl-2 in bladder transitional carcinoma and their significance. Ai. Zheng. 23:181-4.

Wang, K, 2014: Molecular mechanisms of hepatic apoptosis. Cell Death and Disease.5:1-10. Weber, P, Schickinger, S, Wagner, M, et al, 2015: Monitoring of apoptosis in 3D cell cultures by fret and light sheet fluorescence microscopy. Int. J. Mol. Sci. 16:5375-85.

Won-Young, K, Seong Baek, M, Shin Kim, Y, et al, 2016: Glucose-insulin-potassium correlates with hemodynamic improvement in patients with septic myocardial dysfunction. J. Thorac. Dis. 8:3648-57.

Yang, H, Liang, G, Hawkins, B, et al, 2008: Inhalational anesthetics induce cell damage by disruption of intracellular calcium homeostasis with different potencies. Anesthesiology 109: 243-50.

Yingxian, Z, Xiaoyu, X, Guowei, Li, et al, 2017: Isoflurane anesthesia induces liver injury 
by regulating the expression of insulin like growth factor 1. Exp. Therap. Med. 13:1608-13.

Yongxing, S, Cheng, B, Dong, Y, et al, 2014: Time-dependent effects of anesthetic isoflurane on reactive oxygen species levels in HEK-293 Cells. Brain Sci. 4:311-20.

\section{Yosuke, N, Hiroaki, S, Takeshi, O, et al, 2017:}

Glycemia and the cardioprotective effects of insulin pre-conditioning in the isolated rat heart. Cardiovasc. Diabetol. 16:43-50.

Zhang, GH, Dong, YL, Zhang, B, et al, 2008: Isoflurane-induced caspase- 3 activation is dependent on cytosolic calcium and can be attenuated by memantine. J. Neurosci. 28:4551-60.

\section{Explanation of figures}

Fig. 2: Caspase 3 expression in liver sections: $A$ : liver section from GIC showing positive expression of caspase 3 as cytoplasmic \pm membranous brown color in hepatocytes, in sinusoidal lining cells (IHC, DAB, caspase3, x400). B: liver section from GII showing negative expression of caspase 3 in hepatocytes (IHC, DAB, caspase3, x400). C: liver section from GSC showing positive expression of caspase 3 as cytoplasmic \pm membranous color in sinusoidal lining cells (yellow arrow)(IHC, DAB, caspase3,x400). D: liver section from GSI showing negative expression of caspase 3 in hepatocytes (IHC, DAB, caspase3, x400).

Fig. 3: Caspase 7 expression in liver sections: $A$ : liver section from GIC showing positive expression of caspase 7 as cytoplasmic brown color in hepatocytes (IHC, DAB, caspase7, x400). B: liver section from GII showed negative expression of caspase 7 hepatocytes (IHC, DAB, caspase7, x400). C: liver section from GSC showed positive expression of caspase 7 as cytoplasmic color in hepatocytes (red arrow) (IHC, DAB, caspase7, x400). D: liver section from GSI showing negative expression of caspase 7 (IHC, DAB, caspase7, x400).

Fig. 4: Caspase 9 expression in liver sections: $A$ : liver section from GIC showed mild positive expression of caspase 9 as cytoplasmic color in hepatocytes (yellow arrow) (IHC, DAB, caspase9, x400). B: liver section from GII showed negative expression of caspase 9 in hepatocytes (IHC, DAB, caspase9, x400). C: liver section from GSC showed mild positive expression of caspase 9 as cytoplasmic color in hepatocytes (IHC, DAB, caspase9, x400). D: liver section from GSI showed negative expression of caspase 9 in hepatocytes (IHC, DAB, caspase9, $\mathrm{x} 400)$.

Fig. 5: Akt expression in liver sections: A: liver section from GIC showed negative expression of Akt (IHC, DAB, Akt, x400). B: liver section from GII showing positive expression of Akt as cytoplasmic color in hepatocytes (red arrow) (IHC, DAB, Akt, x400).

$C$ : liver section from GSC showed negative expression of Akt (IHC, DAB, Akt, x400). D: liver section from GSI showed positive expression of Akt as cytoplasmic color in hepatocytes (red arrow) (IHC, DAB, Akt, x400).

In GII \& GIC ALT\&AST significantly higher and albumin significantly lower 24hours postoperatively than preoperative levels $(p<0.05)$ Regarding sevoflurane; ALT \& AST in GSC showed significant increase in 24hours postoperative values than preoperative levels ( $<<0.05)$. 24hours postoperative albumin levels in GSC \& GSI significantly lower than preoperative values $(p<0.05)$. Total bilirubin postoperative value significantly lower in GSI compared to preoperative level $(\mathrm{p}<0.05)$.

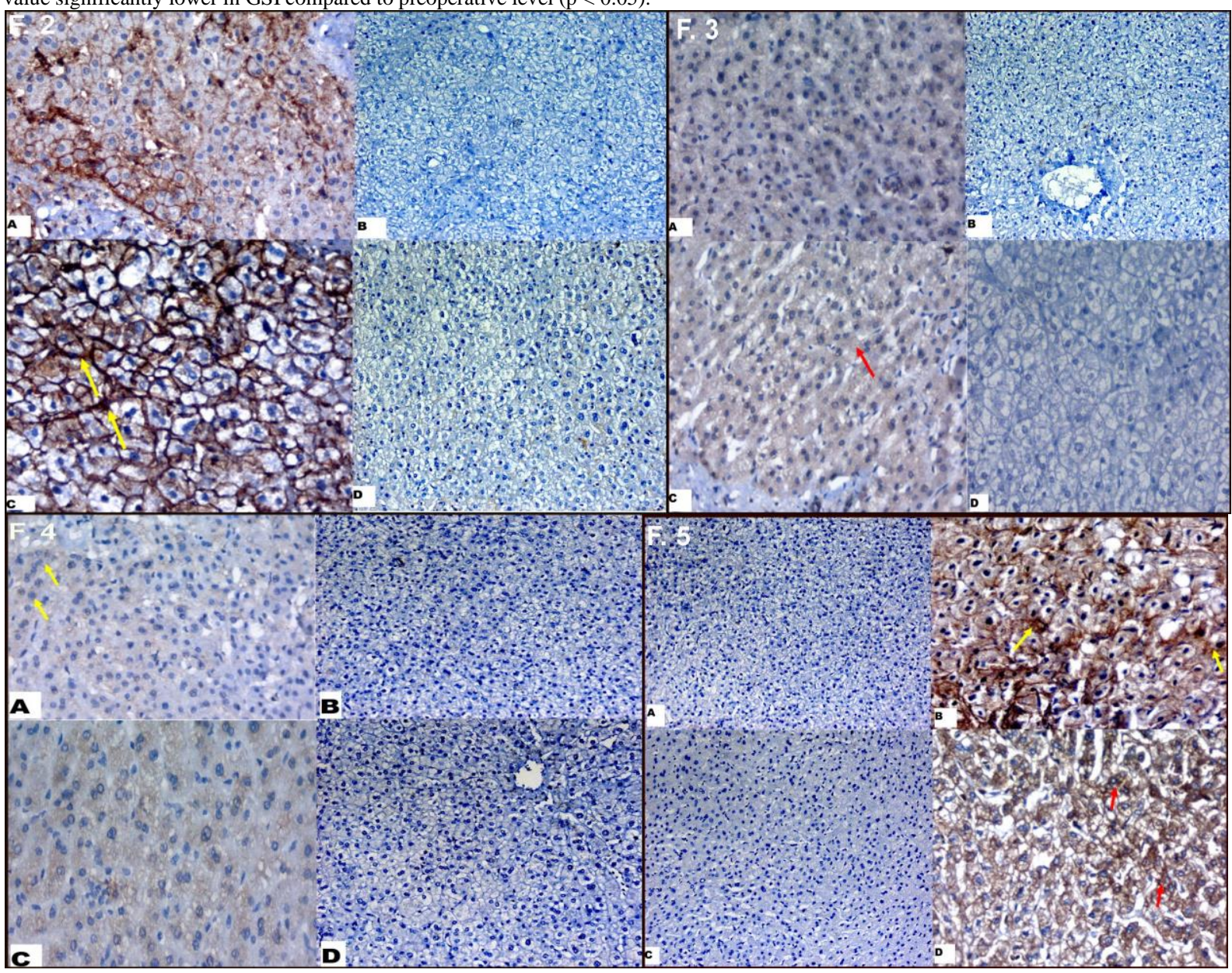

